Comme conclusion de travaux dont l'exposé qui précède ne constitué 'qu'un compte.rendu très sommaire, il est permis d'avancer ce qui suit.

Les différences entre la Truite de ruisseau et la Truite de lac dépendent essentiellement du milieu, spécialement pour ce qui concerne la grosseur.

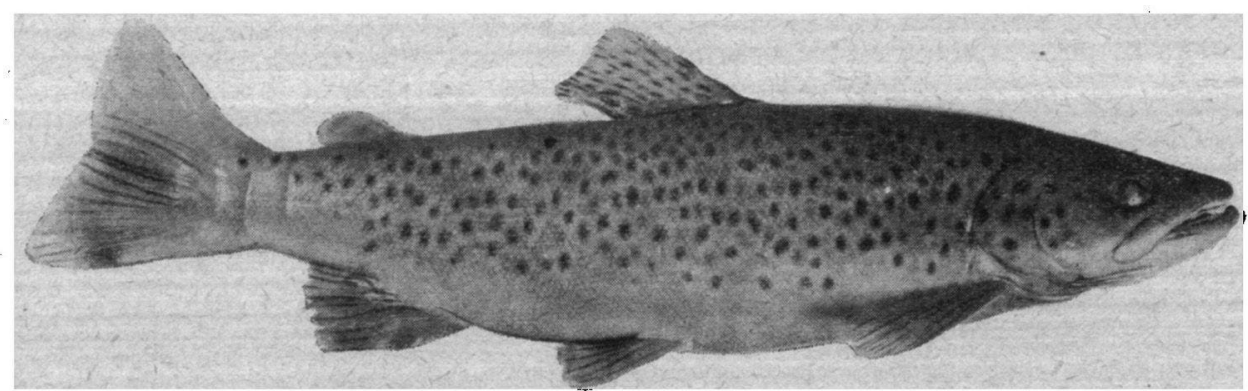

Fro. 10. - Truite de raisseau capturée, à l'age de un été, llans un ruisseau forestier au printemps de 1935, ayant passé son deuxième et son troisième été dans le lac de Stockbergstjärn, où elie rut répéchèe à l'automne de 1996, longue de 36 centimétres. - Réduction au tiers.

Lés petites Truites de ruisseau, même quelque peu âgées, peuvent être, après capture, transportées dans des eaux plus spacieuses et en tirer profit. La progéniture de sujets ayant ainsi amélioré leur condition a, surtout lorsqu'ils sont parvenus à une certaine grosseur, à peu près la même faculté de développement initial rapide que celle issue de la Truite de lac ordinaire.

Il résulte de ces constatations que la Truite de ruisseau est une excellente forme-pour la pratique de la pisciculture et qu'on devrait y recourir, pour l'élevage, beaucoup plus qu'on ne l'a fait jusqu'à ce jour.

\title{
NOTE SUR LA CONFORMATION DE L'OVIDUCTE CHEZ LA CARPE
}

\author{
Par le Docteur Bonis KOSTOMAROV \\ et le Docteur WiLly NOWAK
}

Station d'Lehthyologie et d’Hydrobiologie Franz Harrach, a Velkó-Mézirici (Tchécoslovaquie)

Les recherches sur le développement et l'alimentation de l'alevin de Carpe, que nous avons entreprises depuis plusieurs années, et dont un compte rendu a été publié par le Bulletin français de Pisciculture (I) noụs

(I) Kostomanov, Nowar et Samax : - Recherches biologiques et chimiques sur le développement et l'alimentation de l'alevin de Carpe au cours du premier été. No ro3, Janvier 1937 , p. $165 ;-n^{\circ} 104$, Février 1937 , p. r99.

A la suite de cet article (p. ryg) figure la liste complète des travaux des auteurs sur lo sujet traité. 
cont amené à porter notre attention sur les organes sexuels des géniteurs.

Dès le début de nos travaux, en effet, nous avons dû constater que les organes en question $n$ avaient guère été étudiés jusqu'ici. Le fait avait été déjà signalé par Fiebiger il y a une vingtaine d'années (2). Les auteurs ayant traité de l'anatomie ichthyologique se sont uniquement préoccupés, jusqu'ici, de l'organisation interne de quelques poissons pris comme types ; ce souci d'information générale les a détournés d'entrer dans le détail des particularités spécifiques.

On a complètement perdu de vue que la Carpe a une très grosse importance économique, ce qui eut rendu spécialement désirables, à son égard, des investigations approfondies.

Sans doute Ficbiger, dans son ouvrage précité la concernant, a-t-il rapporté, en les empruntant à ses devanciers, les descriptions fondamentales et résumé les autres ; on doit lui être reconnaissant d'avoir ainsi rassemblé, sur la structure intime du poisson d'étang par excellence, toute la documentation présentant une valeur scientifique, mais il n'a pu lui donner la précision ni, par ailleurs, s'attacher personnellement à combler cette lacune.

L'occasion s'étant présentée, pour nous, d'élucider certaines singularités de l'appareil évacuateur des ovaires chez la Carpe, il nous semble utile de les faire connaître par une courte note.

Avis est donné que les quelques lignes qui suivent concernent, non la glande reproductrice, dont il ne sera pas question, mais seulement le dispositif par lequel, lors de la ponte, les cufs mûrs sont acheminés vers l'orifice sexuel. Rappelons que ce dernier débouche au sommet de la papille génitale, sise entre l'anus et l'orifice uréthral, alors que, chez le mâle, le canal déférent on spermiducte se raccorde, à son extrémilé, à celui de l'organe excréteur, de sorte que laitance et urine ont une issue commune.

Nous nous proposons, sans plus, de donner une description correcte de l'oviducte, sur lequel on ne possède encore, pour la Carpe, que des renseignements sujets à caution.

$A$ en croire Fiebigen, l'enveloppe, de nature conjonctive, qui limite el entoure toute la masse de l'ovaire, disparaîtrait, à l'extrémité postérieure, pour faire place à deux courts oviductes qui se réuniraient en un seul. fort bref, aboutissant derrière l'anus.

Il s'agissait, pour nous, de vérifier l'exactitude des indications ci-dessus. Notre étude a eu, en conséquence, un objectif strictement topographique ; elles ne s'est pas étendue aux organes similaires voisins de l'oviducte, par lesquels sont évacués les excréments et les urines ; elle n'aborde pas l'examen histologique de l'oviducte lui-même ; sa conformation seule a retenu notre intérêt.

(2) Uber den Körperbau des Karpfens. - Osterreichische Fischerei Zeilung, Vienne. I918-1919. 
Le sujet sur lequel portèrent nos observations fut une grosse Carpe mère pesant $1 \mathrm{r}$. $9^{50} \mathrm{~g}$.

On distingue, à la partie postérieure êt inférieure de l'abdomen (Fig. 11)

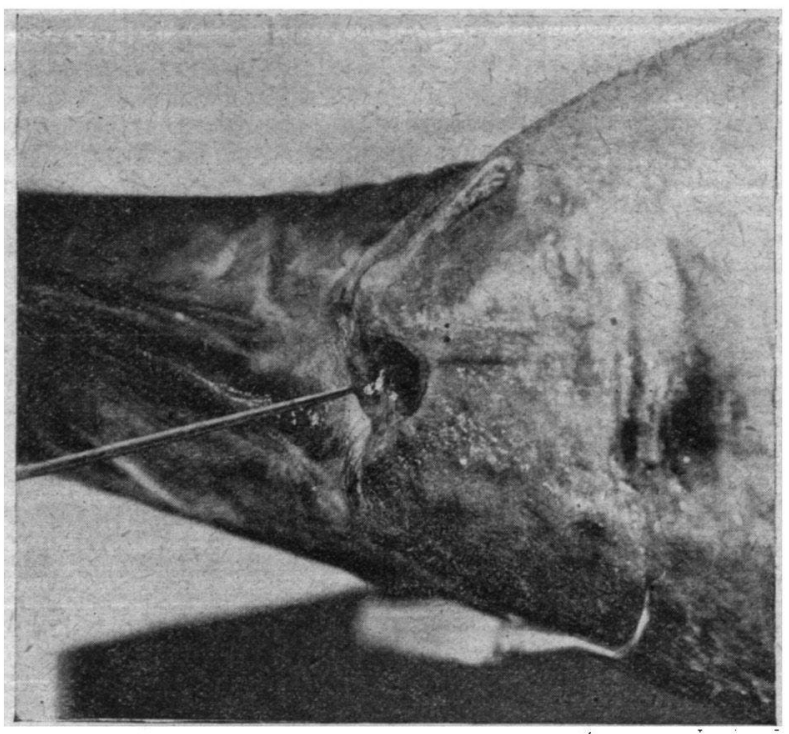

Fia. 11. - Papille génitale de Carpe femelle ávec déoouche de loviducte où est engagó la tête d'une sonde. - A droite, lanus.

l'orifice anal (porus rectalis) et l'orifice sexuel (porus genitalis) ce dernier est celui dans lequel est engagée la tète d'une sonde qui n'a pu être enfoncée profondément pour une raison qui sera exposée plus loin.

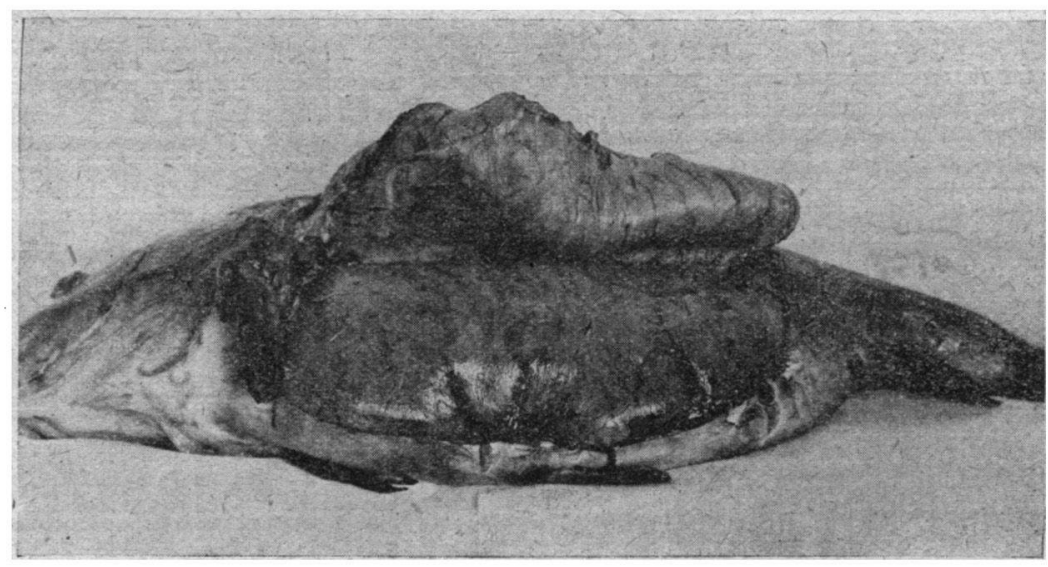

Fig. 12. - Carpe femelle ourerte montrant la masse des ovaires.

Pour étudier l'appareil génital, une incision pratiquée suivant la ligne médiane du ventre et prolongée par deux entailles sur le flanc gauche permit de relever, vers le dos, toute la paroi de la cavité viscérale (Fig. 12). On 
voit immédiatement que cette dernière est presque complètement remplie par les glandes génitales, tant ces dernières sont développées dans la période précédant la ponte; elles ne laissent plus apercevoir l'appareil digestif. On distingue bien les œufs dans leurs follicules, ainsi que les cloisons membraneuses, richement irriguées de vaisseaux sanguins, qui compartimentent la volumineuse masse des ovaires. Tout le système est en liaison par des ligaments suslenteưrs grâce auxquels il est suspendu dans l'abdomen.

Par l'ouverture pratiquée, l'organe sexuel se laisse extraire pour être examiné de plus près (Fiğ. ı3). On remarque alors, qu'à l'arrière, vers

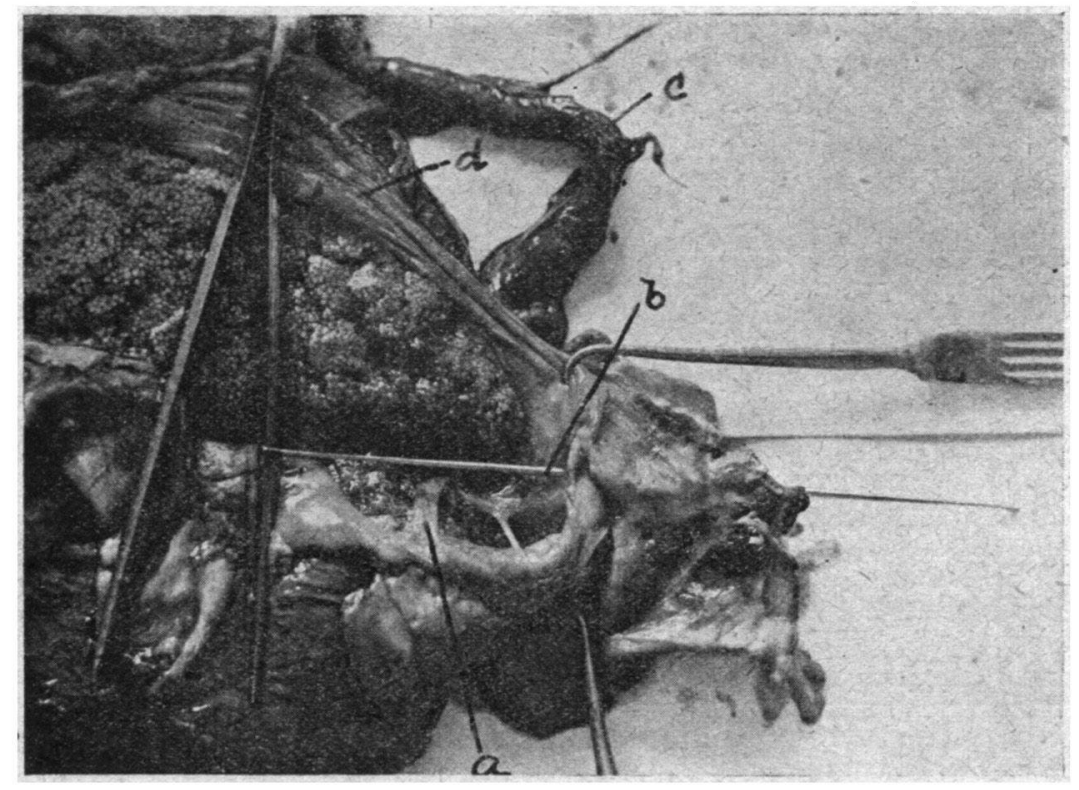

FIG. 13. - Partie arrière de l'ovaire droit de la Carpe.

$a$ : membrane séparative des deux ovaires; $-b$ : oviducte traversé par une sonde ; $-c:$ intestin ; d: membrane enveloppe de l'ovalre.

l'anus, son enveloppe conjonctive s'épaissit progressivement, en s'étirant en pointe. Par contre, la membrane qui s'interpose entre les deux glandes de droite et de gauche, devient lâche, lacuneuse ; finalement le contact s'établit, sauf sur quelques points oì se renforce ce qui subsiste de la cloison séparative. Au delà, le revêtement de l'ovaire, devenu plus résistant, comme il a été dit plus haut, affecte la forme d'une sorte d'entonnoîr, vestibule d'un court oviducte débouchant au sommet de la papille génitale.

Il résulte de nos constatations que Fiebiger a commis une erreur en parlant de deux oviductes confluents. Leur existence n'est pas conciliable avec le fait que les deux ovaires sont en connexion au moins partielle vers leur extrémité postérieure et que leur enveloppe se raccorde avec le canal d'évacuation des œufs par un dispositif en forme de cône. Ce canal est bien visible sur la figure car une sonde le traverse de bout en bout. 
Disons ici que si, avant d'avoir ouvert le poisson, il n'avait pas été possible de pousser quelque peu lia sonde en question, c'est qu'elle se heurtait, chez cet animal relativement âgé, à l'obstacle que constituait, dans l'oviducte, une petite concrétion d'œufs indurés et partiellement résorbés.

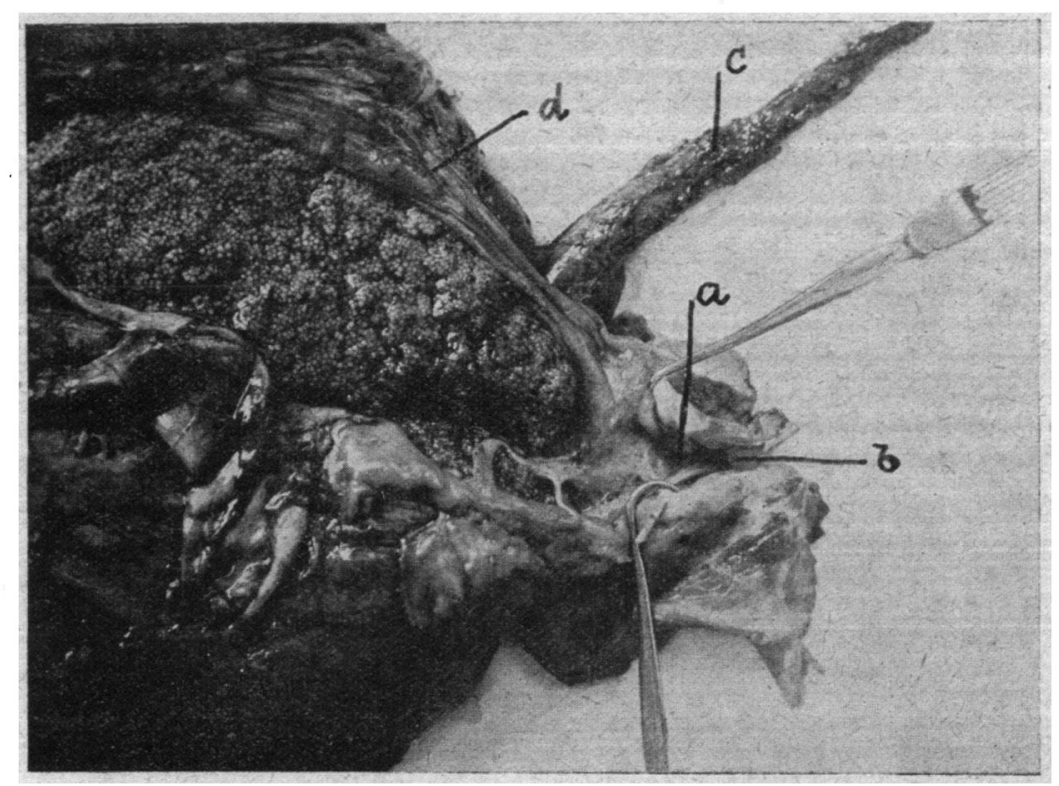

Frg. 14. - Partie arrière dé l'ovaire droit de la Carpe avec l'oviducte fendu de bout en bout. $a$ : entrée de l'oviducte; $-b$ : debouché de l'oviducte au sommet de la papille génitale; $-c:$ intestin $d$ : membrane enveloppe de l'ovaire.

Pour mieux mettre en lumière la disposition de conduit sexuel unique, il a été fondu dans toute sa longueur (Fig. i4). Dans ces conditions l'enIonnoir qui l'amorce se laisse mieux discerner.

Il n'était pas inutile, ce semble, de préciser le détail d'organisation faisaril l'objet de la présente note, les observations āntérieures sur le dispositif d'évacuation des oufs, chez la Carpe, ayant śté superficielles. 